\title{
Application of Square Formulas in the Problems of Research of Automatic Control Systems
}

\author{
Glushkin E.Ya., Kochetkov V.P., Kolovsky A.V. \\ dept. Electrical Power Engineering \\ Khakass Technical Institute, branch of the Siberian Federal University, KTI - branch SFU \\ Abakan, Russia \\ Master8850@mail.ru
}

\begin{abstract}
The article suggests a method of transition from continuous models of automatic control systems (ACS) to discrete ones, based on the application of quadrature formulas. This makes it possible to obtain a model of ACS in the form of a system of linear algebraic equations instead of differential equations. The result of this solution is the discrete values of the ACS state coordinates. That allows one to reduce the expenses of microprocessor time on that part of algorithm of the task which is responsible for the process of calculation of a system condition. The state of the control object and the control law are stored in the controller memory in the form of numeric arrays which is rational when transferring to the digital control of continuous ACS.
\end{abstract}

Keywords-square formulas; automatic control system; digital control; control law

\section{INTRODUCTION}

The paper considers the application of quadrature formulas for the study of continuous automatic control systems (ACS). Most of the methods of analyzing and synthesizing data of the automatic control system are based on the use of an analytical solution of systems of differential equations [1,2]. This is usually a difficult task. Obviously, continuity defines this "difficulties". But the factor of continuity in technical implementation is often not needed $[3,4]$. The principle of continuity is necessary when using analog microchips. Now such systems are rarely used, use microprocessors. Thus, the theory of automatic control tends to the application of discrete mathematics [5].

\section{RELEVANCE}

Technical implementations of the developed control laws are now based on microprocessors. The basis of digital control is the consideration of ACS as a discrete object [6, 7]. For this reason, it is advisable to consider the behavior of a continuous automatic control system as a discrete system. This is usually done by moving to finite differences in the original differential equations. The disadvantage of this approach is that, to increase the accuracy, the least possible value of the finite intervals is required. The second disadvantage is no less important- the error in the previous interval increases the error at all subsequent ones.

The method of analyzing the dynamics of a controlled system, considered in this article, is based on another method of discretization $[8,9]$. Its basis is the application of methods representing the "inverse task" of the theory of quadrature formulas (CF) [10]. This makes it possible to obtain a model of ACS in the form of a system of linear algebraic equations instead of differential equations. The result of this solution is the discrete moments of the ACS state coordinates.

As a result of this application, a transition is made from a system of differential equations to a system of linear algebraic equations. The result of this solution is the discrete values of the ACS state coordinates.

\section{Formulation Of The PROBLEM}

Consider the description of the dynamics of an ACS with control action as $\mathrm{n}$ linear differential equations in the observation interval $\left[t_{1} ; t_{m}\right]$, where

$t_{1}$ - the time point of the initial state of the system

$t_{m}$ - the time moment corresponding to the end point of the phase trajectory

Number equations consecutively. Equation numbers, within parentheses, are to position flush right, as in (1), using a right tab stop. To make your equations more compact, you may use the solidus ( / ), the exp function, or appropriate exponents. Italicize Roman symbols for quantities and variables, but not Greek symbols. Use a long dash rather than a hyphen for a minus sign. Punctuate equations with commas or periods when they are part of a sentence, as in

$$
\left\{\begin{array}{l}
\dot{x}_{1}(t)=a_{1,1} x_{1}(t)+a_{1,2} x_{2}(t)+\ldots+b_{1,1} u_{1}(t)+\ldots+b_{1, l} u_{l}(t) \\
\dot{x}_{2}(t)=a_{2,1} x_{1}(t)+a_{2,2} x_{2}(t)+\ldots+b_{2,1} u_{1}(t)+\ldots+b_{2, l} u_{l}(t) \\
\ldots \\
\dot{x}_{n}(t)=a_{n, 1} x_{1}(t)+a_{n, 2} x_{2}(t)+\ldots+b_{n, 1} u_{1}(t)+\ldots+b_{n, l} u_{l}(t)
\end{array}\right.
$$

In the matrix form, the system looks like

$$
\dot{X}(t)=A X(t)+B U(t)
$$

We take an arbitrary collection of points $\left\{t_{1} ; t_{2} ; \ldots ; t_{m}\right\}$ from this interval and under the solution of the problem of describing the dynamics of the system (1) we will consider the process of obtaining information about the coordinates $x_{i}(t)$ $x_{i}(t)$ at all points $t_{k}$ from the set under consideration, assuming that, as in the classical case, initial conditions, i.e. the value of $x_{i}\left(t_{i}\right)$ is assumed to be known. 
2018 International Conference on Industrial Engineering, Applications and Manufacturing (ICIEAM)

\section{The Solution Of The Problem}

As the basic orthonormal system of functions, we consider the set $\left\{\varphi_{k}(t)\right\}$

$$
\begin{aligned}
& \{\sin (t) ; \cos (t)\} \\
& \{\sin (2 t) ; \cos (2 t)\} \\
& \ldots \\
& \{\sin (k t) ; \cos (k t)\}
\end{aligned}
$$

We multiply each of the equations from the system (1) by one of the functions of the given set, confining ourselves in this set to the value $k \leq n(m-1)$.

We obtain a family of $n(m-1)$ systems

$$
\begin{aligned}
& \left\{\begin{array}{l}
\dot{x}_{1}(t) \phi_{1}(t)=a_{1,1} x_{1}(t) \cdot \phi_{1}(t)+\ldots+b_{1,1} u_{1}(t) \phi_{1}(t)+\ldots+b_{1, l} u_{l}(t) \phi_{1}(t) \\
\dot{x}_{2}(t) \phi_{1}(t)=a_{2,1} x_{1}(t) \phi_{1}(t)+\ldots+b_{2,1} u_{1}(t) \phi_{1}(t)+\ldots+b_{2, l} u_{l}(t) \phi_{1}(t) \\
\ldots \\
\dot{x}_{n}(t) \phi_{1}(t)=a_{n, 1} x_{1}(t) \phi_{1}(t)+\ldots+b_{n, 1} u_{1}(t) \cdot \phi_{1}(t)+\ldots+b_{n, l} u_{l}(t) \phi_{1}(t)
\end{array}\right. \\
& \left\{\begin{array}{l}
\dot{x}_{1}(t) \phi_{2}(t)=a_{1,1} x_{1}(t) \phi_{2}(t)+\ldots+b_{1,1} u_{1}(t) \phi_{2}(t)+\ldots+b_{1, l} u_{l}(t) \phi_{2}(t) \\
\dot{x}_{2}(t) \phi_{2}(t)=a_{2,1} x_{1}(t) \phi_{2}(t)+\ldots+b_{2,1} u_{1}(t) \phi_{2}(t)+\ldots+b_{2, l} u_{l}(t) \phi_{2}(t) \\
\ldots \\
\dot{x}_{n}(t) \phi_{2}(t)=a_{n, 1} x_{1}(t) \phi_{2}(t)+\ldots+b_{n, 1} u_{1}(t) \phi_{2}(t)+\ldots+b_{n, l} u_{l}(t) \phi_{2}(t)
\end{array}\right. \\
& \ldots \\
& \left\{\begin{array}{l}
\dot{x}_{1}(t) \phi_{k}(t)=a_{1,1} x_{1}(t) \phi_{k}(t)+\ldots+b_{1,1} u_{1}(t) \phi_{k}(t)+\ldots+b_{1, l} u_{l}(t) \phi_{k}(t) \\
\dot{x}_{2}(t) \phi_{k}(t)=a_{2,1} x_{1}(t) \phi_{k}(t)+\ldots+b_{2,1} u_{1}(t) \phi_{k}(t)+\ldots+b_{2, l} u_{l}(t) \phi_{k}(t) \\
\ldots \\
\dot{x}_{n}(t) \phi_{k}(t)=a_{n, 1} x_{1}(t) \phi_{k}(t)+\ldots+b_{n, 1} u_{1}(t) \phi_{k}(t)+\ldots+b_{n, l} u_{l}(t) \phi_{k}(t)
\end{array}\right.
\end{aligned}
$$

We integrate the right and left sides of equations (2) on the interval $\left[t_{1} ; t_{m}\right]$

$$
\int_{t_{1}}^{t_{m}} \dot{x}_{i}(t) \phi_{s}(t) d t=\int_{t_{1}}^{t_{m}} \sum_{j=1}^{n} a_{i, j} x_{j}(t) \phi_{s}(t) d t+\int_{t_{1}}^{t_{m}} \sum_{j=1}^{l} b_{i, j} u_{j}(t) \phi_{s}(t) d t
$$

We transform the left side of this expression to the form:

$$
\begin{aligned}
& \int_{t_{1}}^{t_{m}} \dot{x}_{i}(t) \phi_{s}(t) d t=\int_{t_{1}}^{t_{m}}\left(x_{i}(t) \phi_{s}(t)\right)^{\prime} d t-\int_{t_{1}}^{t_{m}} x_{i}(t) \dot{\phi}_{s}(t) d t= \\
& =-x_{i}\left(t_{1}\right) \phi_{s}\left(t_{1}\right)+x_{i}\left(t_{m}\right) \phi_{s}\left(t_{m}\right)-\int_{t_{1}}^{t_{m}} x_{i}(t) \dot{\phi}_{s}(t) d t
\end{aligned},
$$

and the right-hand side of (3), in accordance with the general theory of $\mathrm{CF}$, is replaced (with some approximation) by the sum

$$
\sum_{j=1}^{n} \sum_{k=1}^{m} a_{i, j} c_{k} x_{j}\left(t_{k}\right)\left(t_{k}\right)+\sum_{j=1}^{l} \sum_{k=1}^{m} b_{i, j} c_{k} u_{j}\left(t_{k}\right) \phi_{s}\left(t_{k}\right),
$$

where $c_{k}$ - are the coefficients of the quadrature formula; $x_{j}\left(t_{k}\right)$ - are the coordinates of the system at discrete instants of time $t_{k} ; \varphi_{s}\left(t_{k}\right)-$ is the value of the base function at the point $t_{k} ; u_{j}\left(t_{k}\right)$ - control value at discrete time instants $t_{k}$.

The coefficients of the quadrature formula are found from the exact relations

$$
\int_{t_{1}}^{t_{m}} \phi_{i}(t) d t=\sum_{k=1}^{m} c_{k} \phi_{i}\left(t_{k}\right)
$$

In accordance with these transformations, we write the system of equations (2) in the form:

$$
\begin{aligned}
& -x_{j}\left(t_{1}\right) \phi_{s}\left(t_{1}\right)+x_{j}\left(t_{m}\right) \phi_{s}\left(t_{m}\right)=\sum_{j=1}^{n} \sum_{k=1}^{m} a_{i, j} c_{k} x_{j}\left(t_{k}\right) \phi_{s}\left(t_{k}\right)+ \\
& +\sum_{k=1}^{m} c_{k} x_{j}\left(t_{k}\right) \dot{\phi}_{s}\left(t_{k}\right)+\sum_{j=1}^{l} \sum_{k=1}^{m} b_{i, j} c_{k} u_{j}\left(t_{k}\right) \phi_{i}\left(t_{k}\right)
\end{aligned}
$$

We introduce new notation:

$$
\begin{gathered}
y_{1}=x_{1}\left(t_{1}\right) ; y_{2}=x_{2}\left(t_{1}\right) ; y_{3}=x_{1}\left(t_{2}\right) ; \ldots ; y_{m n}=x_{n}\left(t_{m}\right) ; \\
u_{1}=u_{1}\left(t_{1}\right) ; u_{2}=u_{2}\left(t_{1}\right) ; \ldots ; u_{m}=u_{l}\left(t_{m}\right) ;
\end{gathered}
$$

In these variables, the system of equations (2) in the matrix form will look like this:

$$
\begin{aligned}
& \left(\begin{array}{ccc}
-\phi_{1}\left(t_{1}\right) & 0 \ldots & -\phi_{1}\left(t_{m}\right) \ldots 0 \\
0 & -\phi_{1}\left(t_{1}\right) \ldots & 0 \ldots \phi_{1}\left(t_{m}\right) \ldots \\
\ldots & \ldots & \ldots
\end{array}\right)\left(\begin{array}{c}
y_{1} \\
\ldots \\
y_{m n}
\end{array}\right)= \\
& =\left(\begin{array}{ccc}
c_{1}\left[a_{1,1} \phi_{1}\left(t_{1}\right)+\dot{\phi}_{1}\left(t_{1}\right)\right] & \ldots & c_{m} a_{1, m n} \phi_{1}\left(t_{m}\right) \\
\ldots & \ldots . & \ldots \\
c_{1} a_{n(m-1), 1} \phi_{n}\left(t_{1}\right) & \ldots & c_{m}\left[a_{n(m-1), m n} \phi_{n}\left(t_{m}\right)+\dot{\phi}_{n}\left(t_{m}\right)\right]
\end{array}\right)\left(\begin{array}{c}
y_{1} \\
\ldots \\
y_{m n}
\end{array}\right)+ \\
& +\left(\begin{array}{ccc}
b_{1,1} c_{1} \phi_{1}\left(t_{1}\right) & \ldots & b_{1, m l} c_{m} \phi_{1}\left(t_{m}\right) \\
\ldots & \ldots & \ldots \\
b_{n(m-1), 1} c_{1} \phi_{n}\left(t_{1}\right) & \ldots & b_{n(m-1), m l} c_{m} \phi_{n}\left(t_{m}\right)
\end{array}\right)\left(\begin{array}{c}
u_{1} \\
\ldots \\
u_{m l}
\end{array}\right)
\end{aligned}
$$

We group the variables so that as a result (8) can be written in the form

$$
\left(\begin{array}{ll}
Z_{1} & Z_{2}
\end{array}\right)\left(\begin{array}{l}
Y_{1} \\
Y_{x}
\end{array}\right)=\left(\begin{array}{ll}
D_{1} & D_{2}
\end{array}\right)\left(\begin{array}{l}
Y_{1} \\
Y_{x}
\end{array}\right)+F U
$$

where $Y_{1}=\left(\begin{array}{c}x_{1}\left(t_{1}\right) \\ \ldots \\ x_{n}\left(t_{1}\right)\end{array}\right)-$ is the vector of the initial conditions of the system; $Y_{x}=\left(\begin{array}{c}x_{1}\left(t_{2}\right) \\ \ldots \\ x_{n}\left(t_{m}\right)\end{array}\right)-$ a vector of unknowns; $Z_{1}, Z_{2}$, $D_{1}, D_{2},-$ are submatrices whose dimension is determined by the dimension of the vectors $Y_{1}$ and $Y_{x}$.

The solution of the matrix equation (9) with respect to $Y_{x}$ can be represented in the form:

$$
Y_{x}=\left(Z_{2}-D_{2}\right)^{-1}\left(\left(D_{1}-Z_{1}\right) Y_{1}+F U\right),
$$

This analytic expression is nothing more than a discrete solution of the problem of research the dynamics of the system (1).

\section{Results Of Mathematical Modeling}

For example, we analyze the ACS described by a system of differential equations on the interval $t_{1}=0 ; t_{10}=0.1$ out of ten node points. 
2018 International Conference on Industrial Engineering, Applications and Manufacturing (ICIEAM)

$$
\left\{\begin{array}{l}
\dot{x}_{1}(t)=x_{2}(t)+2 \cdot e^{\frac{t}{10}} \\
\dot{x}_{2}(t)=-x_{1}(t)+3 \cdot e^{\frac{t}{20}}
\end{array}\right.
$$

As initial conditions, we take a point with coordinates $x_{1}(0)$ $=1 ; x_{2}(0)=1$.

Solving the system of equations for a given interval yields the following results

$$
x_{1}(t)=\left[\begin{array}{l}
1 \\
1.04525 \\
1.07568 \\
1.12173 \\
1.15269 \\
1.18386 \\
1.21523 \\
1.24679 \\
1.27856 \\
1.31052
\end{array}\right] ; x_{2}(t)=\left[\begin{array}{l}
1 \\
1.02968 \\
1.0491 \\
1.0777 \\
1.09639 \\
1.11479 \\
1.1329 \\
1.1507 \\
1.1682 \\
1.1854
\end{array}\right] .
$$

Using the above method of determining the position of the system at discrete instants of time $\left[t_{1} ; t_{2} ; \ldots ; t_{10}\right]$, we obtain the following values.

$$
x_{p 1}(t)=\left[\begin{array}{l}
1 \\
1.03111 \\
1.09877 \\
1.15155 \\
1.17588 \\
1.20541 \\
1.23393 \\
1.26159 \\
1.28397 \\
1.31052
\end{array}\right] ; x_{p 2}(t)=\left[\begin{array}{l}
1 \\
1.02655 \\
1.05616 \\
1.08459 \\
1.10436 \\
1.11949 \\
1.13816 \\
1.15469 \\
1.17051 \\
1.1854
\end{array}\right] .
$$

Comparing the results calculated using this method and the classical method, we obtain the relative error in percent

$$
E_{x 1}=\left[\begin{array}{l}
0 \\
1.353 \\
2.147 \\
2.658 \\
2.012 \\
1.82 \\
1.539 \\
1.187 \\
0.423 \\
0
\end{array}\right] ; E_{x 2}=\left[\begin{array}{l}
0 \\
0.304 \\
0.673 \\
0.639 \\
0.727 \\
0.422 \\
0.464 \\
0.347 \\
0.198 \\
0
\end{array}\right] .
$$

The calculation results in the package Mathematica [11] showed that the relative error of the proposed method does not exceed $3 \%$ in comparison with the classical method of calculation.

\section{PRACTiCAL SignificANCE}

In most cases, the practical implementation of automation tasks for objects whose mathematical model in the original version uses systems of linear continuous equations of the form (1), digital control is used. The transition from the continuous description to the proposed one allows to reduce the costs of microprocessor time for that part of the task algorithm that is responsible for the process of calculating the state of the system.

This is achieved due to the fact that the state of the control object and the control law are stored in the controller memory in the form of numeric arrays, which is rational when transferring to digital control of continuous ACS.

\section{CONCLUSION}

A method is proposed for obtaining discrete mathematical models, which is based on the use of quadrature formulas representing an integral notation for systems of linear continuous equations. Using the expansion in a given orthonormal system of functions, we obtain a numerical matrix equation as an example of a discrete representation of the original system.

\section{REFERENCES}

[1] N.N. Krasovskiy, The theory of motion control. Moscow: Science, 1967.

[2] L. Tie and J.-S. Li A, "On controllability of discrete-time linear ensemble systems with linear parameter variation," Proc. of the American Control Conf., pp. 6357-6362, 2016.

[3] J. Li, X. Wang, F. Han, and G. Wei, "Fault detection for discrete piecewise linear systems with infinite distributed time-delays" Int. Journal of Systems Science, no. 48(16), pp. 3431-3439, 2017.

[4] V. Gaitsgory, A. Parkinson, and I. Shvartsman, "Linear programming formulations of deterministic infinite horizon optimal control problems in discrete time," Discrete and Continuous Dynamical Systems - Series B, no. 22(10), pp. 3821-3838, 2017.

[5] D. Zeng, K.-T. Wu, Y. Liu, R. Zhang, and S. Zhong, "Event-triggered sampling control for exponential synchronization of chaotic Lur'e systems with time-varying communication delays," Nonlinear Dynamics, pp. 1-17, 2017.

[6] D. Rotondo, F. Nejjari, and V. Puig, "Shifting finite time stability and boundedness design for continuous-time LPV systems," Proc. of the American Control Conf., pp. 838-843, 2015.

[7] M.K. Kabidoldanov, "On the Control of Linear Discrete Systems," The problems of automation and contro, no. 1(28), pp. 36-39, 2015.

[8] D.P. Kim, "Algebraic method of synthesis of discrete control systems," Mechatronics, Automation, Control, no. 4, pp. 8-13, 2013.

[9] S.P. Zubova and Thanh Tuan Chan, "On one method of constructing control and states for a discrete stationary control system," Bulletin of Voronezh State University. Series: Economics and Control, no. 2, pp. $162-168,2010$.

[10] G. Bateman and A. Erdei, Higher transcendental functions. Moscow: Science, 1974.

[11] V. Dyakonov, Mathematica 4. St. Petersburg: Peter, 2001. 